\title{
RhoA regulates resistance to irinotecan by regulating membrane transporter and apoptosis signaling in colorectal cancer
}

\author{
Huang Ruihua ${ }^{1}$, Zhang Mengyi ${ }^{1}$, Zhao Chong ${ }^{2}$, Qiu Meng ${ }^{1}$, Ma Xin ${ }^{1}$, Tang Qiulin ${ }^{1}$, \\ Bi Feng ${ }^{1}$, Liu Ming ${ }^{1}$ \\ ${ }^{1}$ Department of Medical Oncology/Laboratory of Signal Transduction and Molecular Targeted Therapy, West China Hospital, \\ Sichuan University, Chengdu, Sichuan Province, China \\ ${ }^{2}$ Department of Radiotherapy, The Tumor Hospital of Chengdu/The Seventh Peoples's Hospital of Chengdu, Chengdu, Sichuan \\ Province, China
}

Correspondence to: Liu Ming, email: mingliu721@aliyun.com

Keywords: irinotecan, RhoA, colorectal cancer, chemoresistance

Received: March 09, $2016 \quad$ Accepted: November 08, 2016

Published: November 24, 2016

\section{ABSTRACT}

Colorectal cancer (CRC) is a major cause of mortality and morbidity worldwide. While surgery remains the mainstay of treatment in early stage CRC, chemotherapy is usually given to prolong the overall survival and improve the quality of life for metastatic colorectal cancer ( $\mathrm{MCRC}$ ). But drug resistance is one of the major hurdles of mCRC treatment, and the underlying mechanisms are still largely unknown. In this study, we show that, compared with parental cells, RhoA is up-regulated in irinotecan (CPT-11)-resistant CRC cells. Furthermore, inhibition of RhoA in drug resistant cells, at least partially, rescues the resistance against irinotecan and increases the sensitivity to other chemotherapeutic drug by inhibiting expression of MDR1, MRP1and GSTP1, promotes apoptosis by suppressing the expression of $\mathrm{BCL}-\mathrm{XL}$ and $\mathrm{Bcl}-2$ and increasing Bax expression, and significantly decreases side population cells. Our results suggest that, in addition to survival, proliferation, migration, adhesion, cell cycle and gene transcription, RhoA is also involved in chemoresistance by regulating the expression of membrane transporter and apoptosis protein in colorectal cancer. They raise an interesting possibility that the expression of RhoA may indicate a poor prognosis due to the high probability to therapy resistance and, on the other hand, inhibition of RhoA activity and function may overcome chemoresistance and improve the effectiveness of clinical treatment of CRC.

\section{INTRODUCTION}

Colorectal cancer is one of the most malignant cancers of digestive system, and its morbidity is increasing with years. The main treatment at present is surgeryplus chemotherapy-based comprehensive therapy. Irinotecan is one of common chemotherapeutic drugs for colorectal cancer, which inhibits DNA synthesis of cancer cells through inhibition of Topo I activity [1-3]. At present, FOLFIRI regimen, including CPT-11, 5-Fu and leucovorin, was considered as one of the standard first line or second line treatment of mCRC [4]. However, most patients become resistant to chemotherapy after a couple of cycles, leading to the failure of treatment. Therefore, chemoresistance is a bottleneck constraint of current therapies and the effectiveness has been seriously hindered. The mechanisms of chemoresistance in cancer cells are complicated and main mechanisms include (1) increased drug efflux mediated by membrane transport proteins such as $\mathrm{ABC}$ transport proteins family members p-glycoprotein (p-gp), multidrug resistance-associated protein (MRP), and breast cancer resistance protein (BCRP/ABCG2) $[5,6,12]$; (2) reduction in cell apoptosis mediated by bcl-2 family [7-9], NF-kB, and p53 [10]; and (3) other drug resistance mechanisms mediated by topoisomerase and glutathione S-transferase (GST). The reduction of topoisomerase activity leads to decreased affinity of topoisomerase with its target cells, which rendering target cells resistant to chemotherapy [11].

Rho GTPases family is an important intracellular signaling module and plays a key role in cell survival, proliferation, adhesion, cell cycle, gene transcription 
and other aspects of cell activities. While a subset of Rho GTPases are constitutively active, the majority of Rho family members act as molecular switches, cycling between the active, GTP-bound form and the inactive, GDP-bound form that are controlled by guanine nucleotide exchange factors (GEFs), GTPase-activating proteins (GAPs) and GDP-dissociation inhibitors (GDIs) [13]. As a primary Rho GTPase, RhoA is involved in multiple aspects of tumor development and progression, such as proliferation, migration, invasion, metastasis, and apoptosis $[14,15]$. However, whether and how RhoA regulates drug resistance in colorectal cancer are poorly understood.

CPT-11 is one of the key chemotherapy agents for $\mathrm{mCRC}$ and resistance to CPT-11 is a major reason for the treatment failure. We established the CPT-11-resistant cells by exposure to a low dose of CPT-11 in culture media for 12 months, and preliminary results of comparative proteomic analysis of CPT-11-resistant CRC cells and parental cells show that irinotecan resistance is related to changes in several pathways such as cell proliferation/ differentiation, cell apoptosis, and electron transport/ redox regulation $[16,17]$. In this study, we have explored the role of RhoA on CPT-11 resistance and underlying molecular mechanisms. Our study has provided a novel insight into the molecular mechanisms of drug resistance in colorectal cancer, and suggested that targeting RhoA signaling might improve the effectiveness of clinical treatment of colorectal cancer.

\section{RESULTS}

\section{RhoA expression is increased significantly in CPT-11-resistant CRC cells}

To determine if RhoA is involved in chemoresistance in colorectal cancer, we first examined the expression of RhoA in CPT-11-resistant colorectal cancer cells (SW620/ CPT-11 and LoVo/CPT-11) and parental cells. Total mRNAs and proteins of SW620/CPT-11, LoVo/CPT-11 and its parental cells were extracted and the expression of RhoA mRNA and protein were analyzed by qRT-PCR and Western blot, respectively. The results showed that RhoA mRNA and protein expression levels were higher in SW620/CPT-11 and LoVo/CPT-11 than in parental cells (Figure 1). These results suggest a possible relationship between RhoA and chemoresistance in CRC.

\section{Inhibition of RhoA expression, at least partially, rescues CPT-11 chemoresistance of CRC cells}

To examine if the increased RhoA expression is important for chemoresistance of CRC, we transfected SW620/CPT-11, LoVo/CPT-11 and parental cells with siRNA targeting RhoA coding sequences (si-RhoA) and control siRNA oligo (si-control), and then examined drug resistance index of the cells to oxaliplatin (L-OHP), cisplatin(DDP), Irinotecan (CPT-11), paclitaxel (PTX), 5-FU, epirubicin (EPI), and Etoposide (VP-16), by in vitro drug sensitivity assay. As shown in Table 1, the IC50 of all of the 7 chemotherapy drugs in CPT11-resistant CRC cells was significantly increased compared to that in parental cells. However, the IC50 was significantly decreased upon transfection of si-RhoA $(p<0.05)$. These data suggest that inhibition of RhoA expression in CPT-11-resistant CRC cells, at least partially, overcomes chemoresistance of CRC.

\section{Inhibition of RhoA suppresses the expression of P-gp, MRP1 and GSTP1 in CPT-11-resistant CRC cells}

To explore the mechanisms underlying RhoAregulated chemoreistance, Western blot was performed to determine the expression of p-glycoprotein(P-gp) and multidrug resistance-associated protein 1(MRP1), two membrane transport proteins known to mediate drug efflux, in CPT-11-resistant CRC cells and parental cells. As expected, P-gp and MRP1 were up-regulated in CPT11-resistant CRC cells (Figure 2). Next, to test whether P-gp and MRP1 are regulated by RhoA, SW620/CPT-11 and LoVo/CPT-11 cells were transfected with si-RhoA or control, and expression of P-gp and MRP1 were examined 48 hours after transfection. Interestingly, inhibition of RhoA by si-RhoA suppressed P-gp (Figure 2A-2C) and MRP1(Figure 2A-2C) expression in CPT-11-resistant CRC cells.

In addition to membrane transporting proteins, glutathione S-transferase(GST) is also involved in drug resistance [18]. The expression of GSTP1, an important member of GST family, was determined by Western blot in CPT-11-resistant CRC cells and parental cells. As shown in Figure 2, GSTP1 was up-regulated in CPT-11resistant CRC cells and inhibition of RhoA by si-RhoA suppressed GSTP1 expression (Figure 2A-2C). These data suggest that both of membrane transport proteins and GST are regulated by RhoA, which, at least partially, contribute to CPT-11 resistance of CRC cells.

\section{Inhibition of RhoA induces apoptosis in CPT-11- resistant CRC cells}

To further understand the mechanisms of RhoA in the regulation of chemoresistance of CRC cells, we investigated the effects of RhoA on apoptosis in CPT-11-resistant CRC cells, as evasion of apoptosis is a crucial event during the process of chemoresistance. We found that, compared with parental cells, CPT-11-resistant CRC cells showed a significant decrease in apoptosis rate, as determined by Annexin V-FITC/PI staining (Figure 3A-3C), whereas inhibition of RhoA by si-RhoA resulted in a significant increase in apoptosis rate in CPT-11-resistant 
Table 1: Inhibition of RhoA expression, at least partially, rescues chemoresistance of CRC cells

\begin{tabular}{llllllll}
\hline & \multicolumn{1}{c}{ CPT-11 } & \multicolumn{1}{c}{ 5-FU } & \multicolumn{1}{c}{ PTX } & \multicolumn{1}{c}{ DDP } & \multicolumn{1}{c}{ EPI } & \multicolumn{1}{c}{ OHP } & \multicolumn{1}{c}{ VP16 } \\
\hline SW620 & $0.58 \pm 0.04$ & $0.50 \pm 0.08$ & $0.15 \pm 0.02$ & $0.12 \pm 0.05$ & $0.42 \pm 0.04$ & $1.07 \pm 0.15$ & $0.15 \pm 0.12$ \\
SW620/CPT-11 & $12.91 \pm 0.79^{*}$ & $1.31 \pm 0.21^{*}$ & $1.17 \pm 0.20^{*}$ & $0.79 \pm 0.35^{*}$ & $2.33 \pm 0.10^{*}$ & $4.08 \pm 0.06^{*}$ & $1.87 \pm 0.26^{*}$ \\
Si-control & $12.31 \pm 0.89$ & $1.23 \pm 0.21$ & $1.23 \pm 0.13$ & $0.77 \pm 0.25$ & $2.04 \pm 0.04$ & $4.04 \pm 0.04$ & $1.84 \pm 0.21$ \\
Si-RhoA & $3.68 \pm 0.17^{*}$ & $0.87 \pm 0.06^{*}$ & $0.35 \pm 0.04^{*}$ & $0.23 \pm 0.03^{*}$ & $0.46 \pm 0.01 *$ & $1.9 \pm 0.07 *$ & $0.29 \pm 0.03^{*}$ \\
LoVo & $1.83 \pm 0.33$ & $0.24 \pm 0.03$ & $0.14 \pm 0.03$ & $0.11 \pm 0.02$ & $0.35 \pm 0.05$ & $0.57 \pm 0.04$ & $0.24 \pm 0.03$ \\
LoVo/CPT-11 & $15.57 \pm 1.43^{*}$ & $6.04 \pm 0.54^{*}$ & $1.07 \pm 0.22^{*}$ & $1.47 \pm 0.09^{*}$ & $1.45 \pm 0.49^{*}$ & $3.27 \pm 0.32^{*}$ & $1.50 \pm 0.29^{*}$ \\
Si-control & $14.95 \pm 0.10$ & $5.39 \pm 0.67$ & $1.07 \pm 0.03$ & $1.55 \pm 0.15$ & $1.22 \pm 0.32$ & $3.18 \pm 0.21$ & $1.51 \pm 0.22$ \\
Si-RhoA & $4.69 \pm 0.30^{*}$ & $0.30 \pm 0.01^{*}$ & $0.74 \pm 0.06^{*}$ & $0.44 \pm 0.02^{*}$ & $0.72 \pm 0.10^{*}$ & $0.55 \pm 0.10^{*}$ & $0.29 \pm 0.04^{*}$ \\
\hline
\end{tabular}

SW620/CPT-11, LoVo/CPT-11 and parental cells were transfected with si-RhoA or si-control, and then drug resistance to L-OHP, DDP, CPT-11, PTX, 5-FU, EPI and VP-16 was tested by in vitro drug sensitivity assay. Data are representative of three independent experiments performed in triplicate. *indicates a significant difference from control oligo-transfected cells $(p<0.05)$.
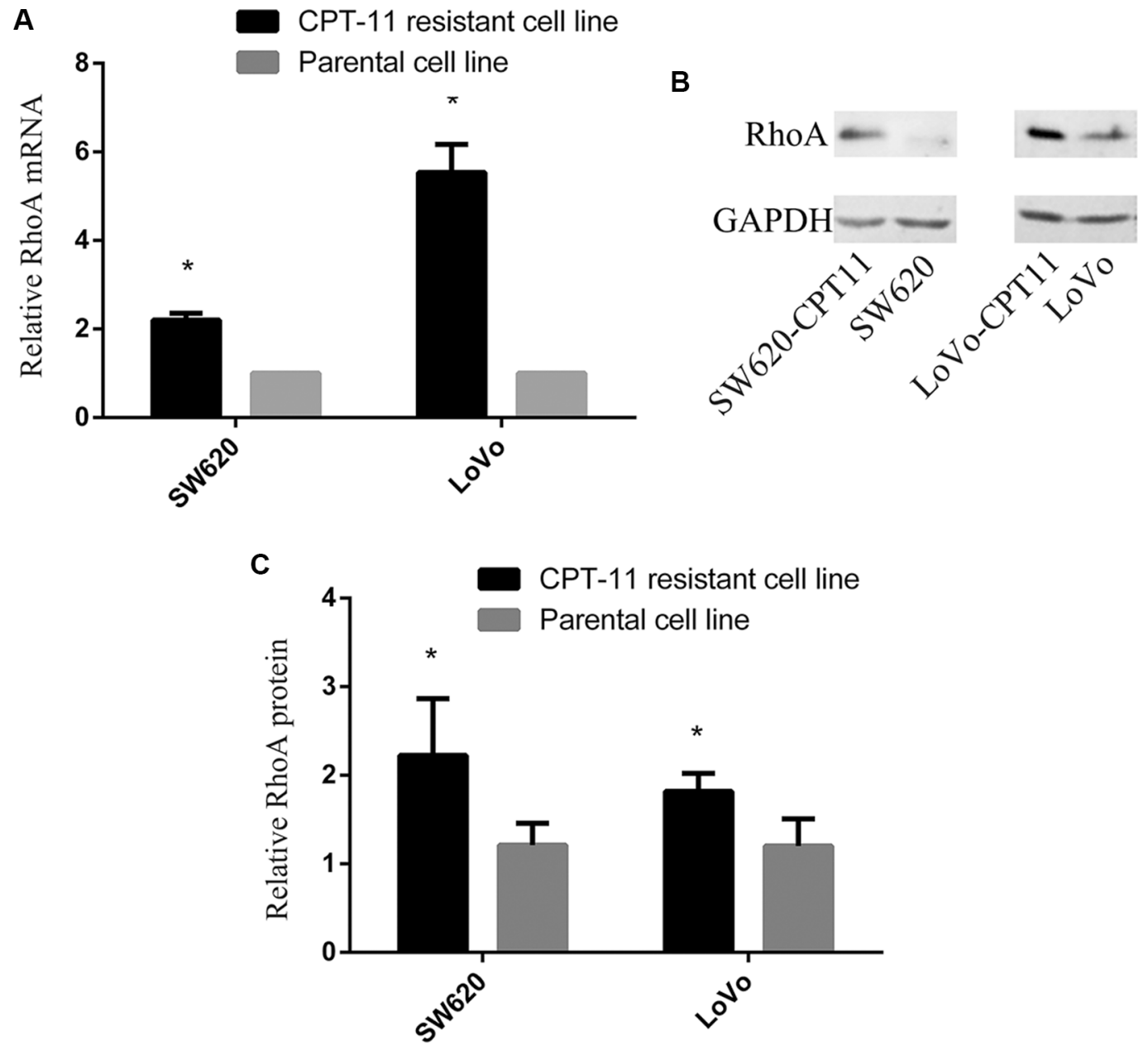

Figure 1: RhoA expression is increased significantly in CPT-11-resistant CRC cells. (A) qRT-PCR analysis of RhoA mRNA expression in CPT-11-resistant cells and parental cells. RhoA mRNA expression was normalized to GAPDH mRNA expression, and data

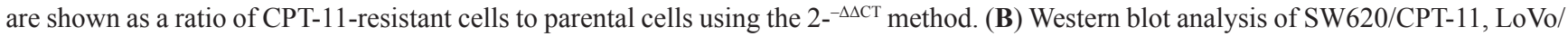
CPT-11 and parental cells for RhoA protein expression. (C) The band intensities in B were quantified with the ODYSSEY Infrared Imaging System (LI-COR Biosciences). Data represent the mean \pm S.D. from three independent experiments. *indicates $p<0.05$. 
CRC cells (Figure 3A-3C). Similar results were obtained by TUNEL assay (Figure 4A-4C). These results suggest that inhibition of RhoA induces apoptosis in CPT-11resistant CRC cells.

To characterize the mechanistic role of RhoA in apoptosis in CPT-11-resistant CRC cells, Western blot was performed to determine the protein expression of bcl2 family members in CPT-11-resistant CRC cells and parental cells. As shown in Figure 5, pro-apoptotic bax was down-regulated, and anti-apoptotic Bcl-xl and $\mathrm{Bcl} 2$ were up-regulated in CPT-11-resistant CRC cells. To determine whether bax, $\mathrm{Bcl}-\mathrm{xl}$ and $\mathrm{Bcl} 2$ are regulated by RhoA, SW620/CPT-11 and LoVo/CPT-11 cells were transfected with si-RhoA, and expression of bax, Bcl-xl, and $\mathrm{Bcl} 2$ were examined by Western blot 48 hours after transfection. Interestingly, inhibition of RhoA by siRhoA suppressed the expression of Bcl-xl and $\mathrm{Bcl} 2$, and promoted the expression of bax in CPT-11-resistant CRC cells (Figure 5A-5C). These results suggest that CPT-11 chemoresistance of $\mathrm{CRC}$ cells could be attributed to the decreased apoptosis that is regulated by RhoA through regulating Bcl-2 signaling.

\section{Inhibition of RhoA decreases the proportion of SP cells in CPT-11-resistant CRC cells}

Several studies have demonstrated that isolated side population (SP) cells from solid tumors exhibit cancer stem cell-like properties, and are responsible for drug resistance during chemotherapy and tumor recurrence [19]. To investigate the role of SP cells in CPT-11 resistance of CRC cells, and the role of RhoA in SP cells in CPT-11resistant CRC cells, we detected the proportion of SP cells using the Hoechst/33342 dye exclusion technique in CPT11-resistant CRC cells and parental cells. Compared with parental cells, the proportion of SP cells were increased significantly in both of the CPT-11-resistant CRC cells (SW620-CPT11,LoVo-CPT11) $(p<0.05$, Figure 6A-6C), whereas suppression of RhoA led to a drastic decrease of SP cells in CPT-11-resistant CRC cells $(p<0.05$,
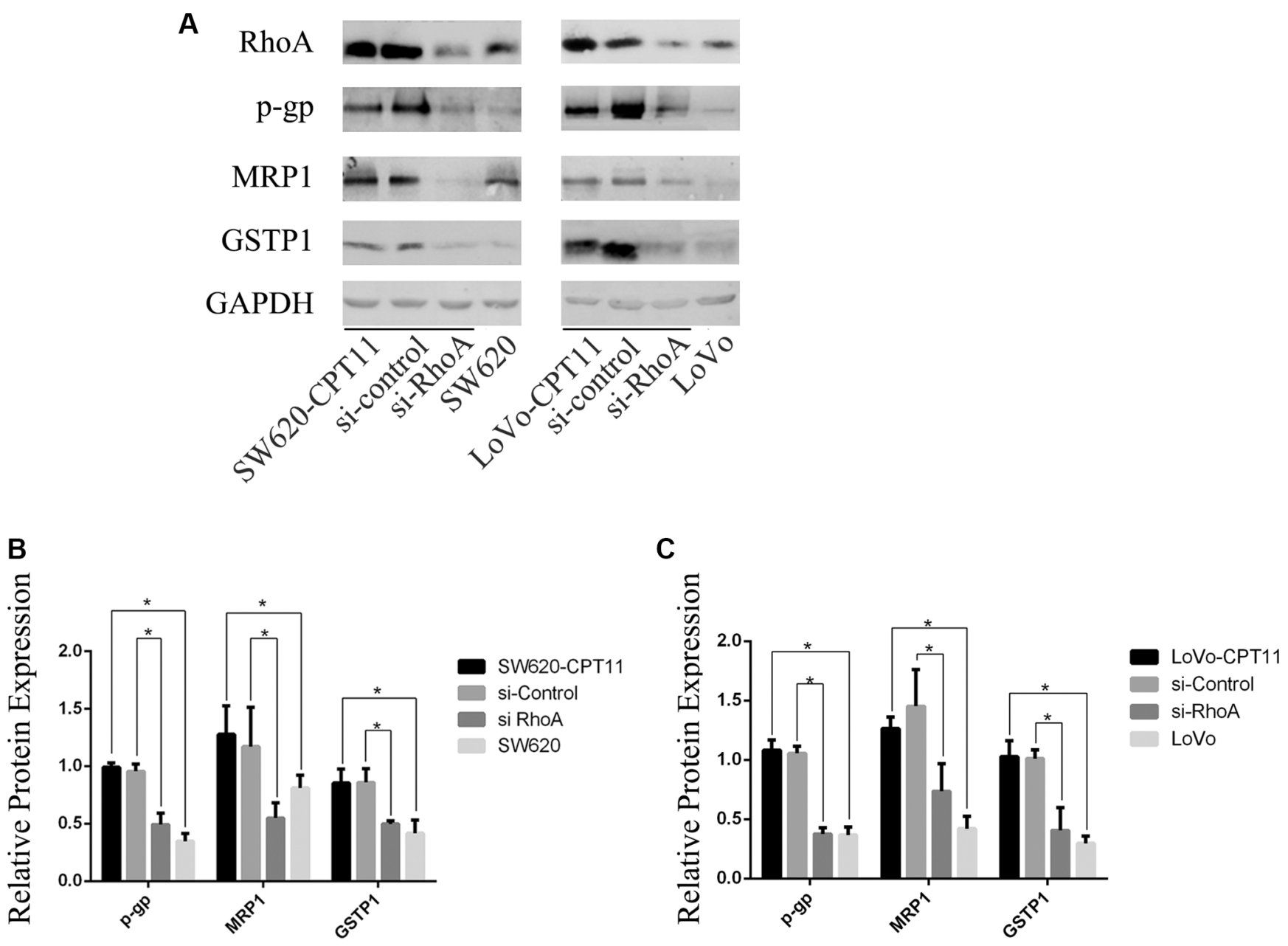

Figure 2: Inhibition of RhoA suppresses the expression of P-gp, MRP1 and GSTP1 in CPT-11-resistant CRC cells. (A) Western blot analysis of CPT-11-resistant CRC cells (SW620/CPT-11, LoVo/CPT-11), CPT-11-resistant CRC cells transfected with si-control or si-RhoA, and parental cells for RhoA, P-gp , MRP1 and GSTP1 protein expression. (B) and (C) The band intensities in A were quantified with the ODYSSEY Infrared Imaging System (LI-COR Biosciences). Data represent the mean \pm S.D. from three independent experiments. *indicates $p<0.05$. 
Figure 6A-6C). These data suggest that RhoA-induced SP cells contribute to drug resistance of CRC cells.

\section{DISCUSSION}

CRC is a major cause of mortality and morbidity worldwide [1]. For the advanced CRC, chemotherapy is recommended as a major treatment. But drug resistance highly limits therapeutic options and its molecular mechanism needs to be understood urgently. As a key member of the Rho GTPase family, RhoA is known to be involved in proliferation, migration, invasion, apoptosis, and angiogenesis, in several types of cancers [13-15].
However, the role of RhoA in chemoresistance is poorly defined. In this study, we investigated the effects of RhoA on CPT-11 resistance and underlying molecular mechanisms in detail. The results suggest that increased expression of RhoA promotes resistance to irinotecan by regulating membrane transporter and apoptosis signaling in CPT-11-resistanct CRC cells. Our findings are in line with that by Carloni $\mathrm{V}$ who has shown that RhoA is involved in cell fusion and causes the appearance of cells resistant to both 5-fluorouracil and oxaliplatin in a metastatic model of colon carcinoma [28].Thus, the expression of RhoA may indicate a poor prognosis due to the high probability to therapy resistance.
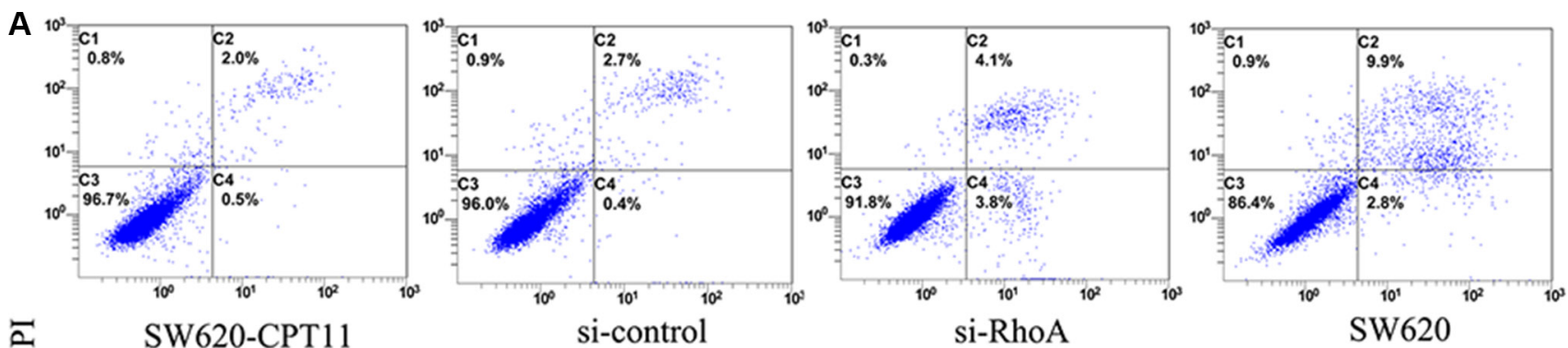

$\overparen{1.1}$

SW620-CPT11

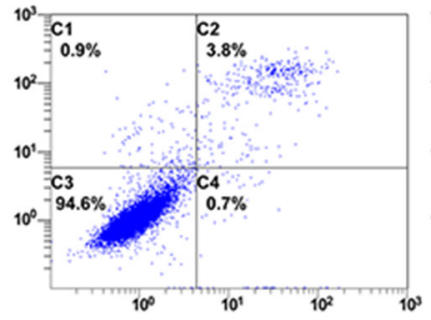

LoVo-CPT11
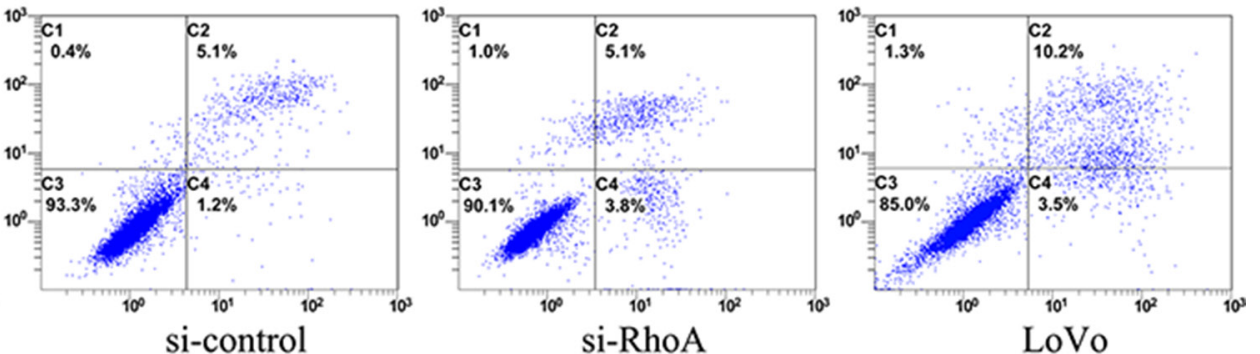

FITC
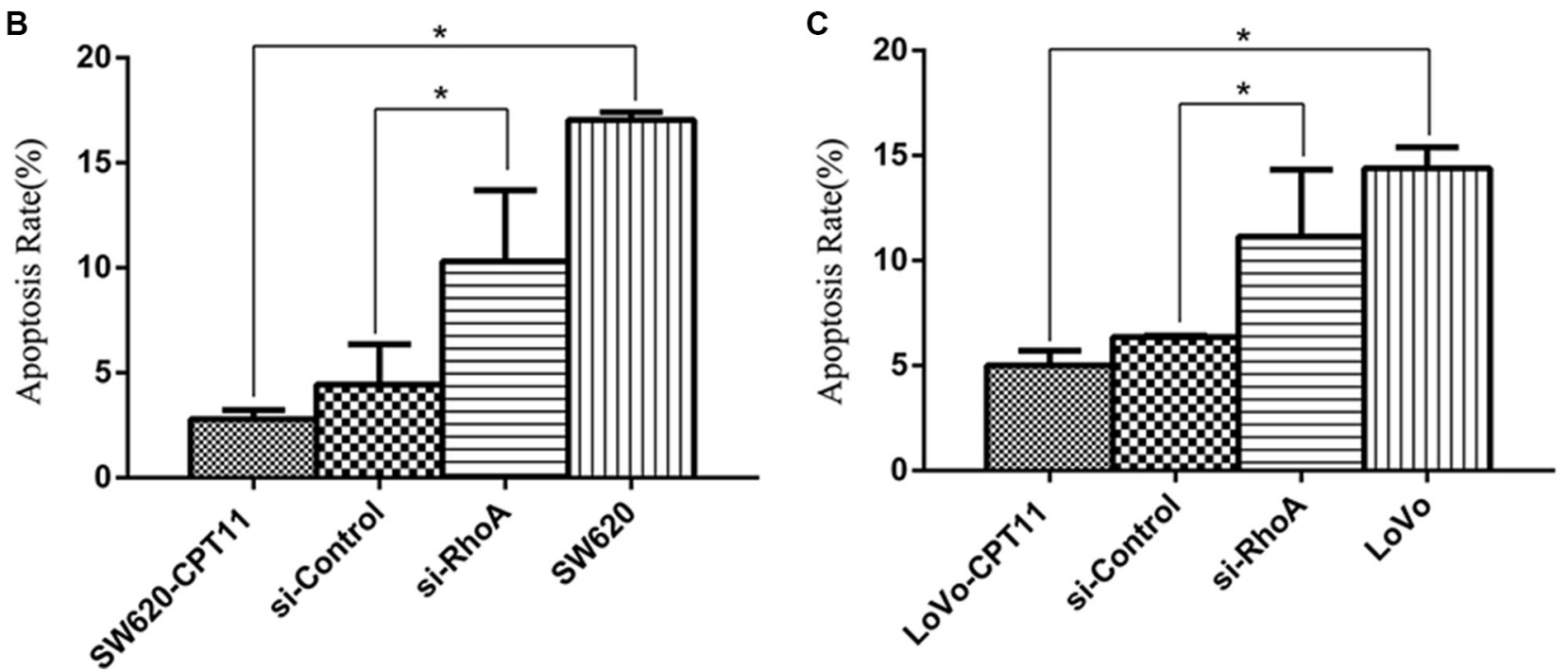

Figure 3: Inhibition of RhoA induces apoptosis in CPT-11-resistant CRC cells by FACS analysis. (A) The apoptosis rates of CPT-11-resistant CRC cells (SW620/CPT-11 or LoVo/CPT-11), CPT-11-resistant CRC cells transfected with si-control or si-RhoA, and parental cells were determined by FACS analysis. (B) and (C) Apoptosis rate of CRC cells in A was quantified. Data are representative of three independent experiments. *indicates $p<0.05$. 
ABC transporters transport a wide range of substrates including metabolic products, nutrients, lipids, and drugs across extracellular and intracellular membranes. $\mathrm{ABC}$ transporters are well-known to be associated with chemoresistance. Expression of various ABC transporters is increased following chemotherapy, causing a reduction in the intracellular accumulation of chemotherapy drugs, and impacting on mCRC survival [12].
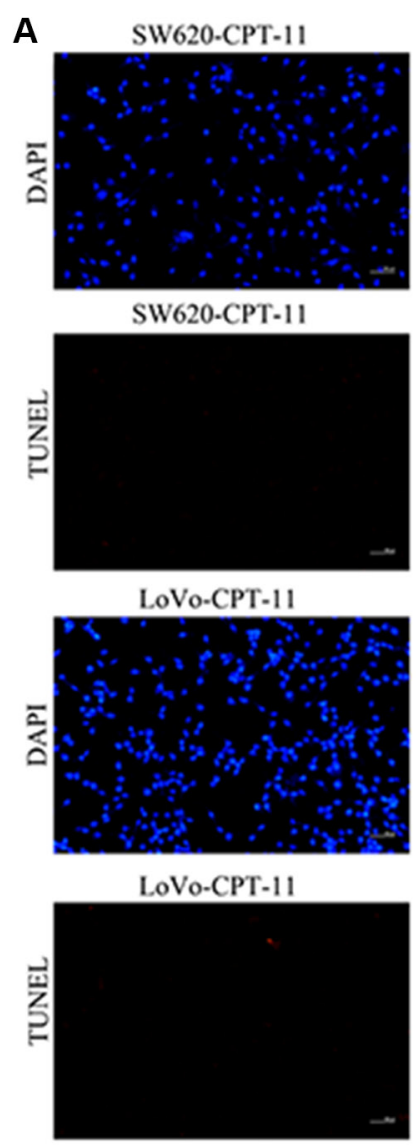

B

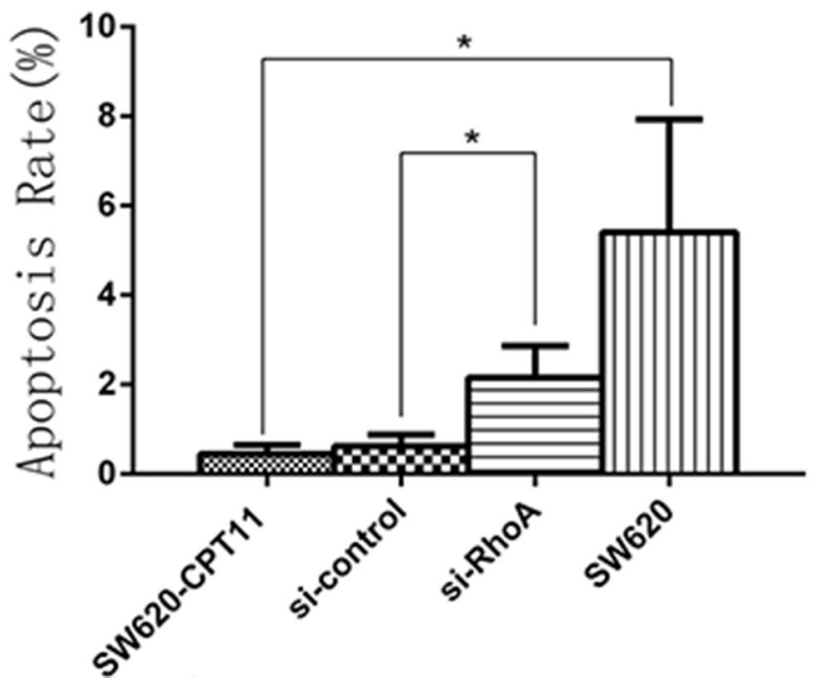

Si-control

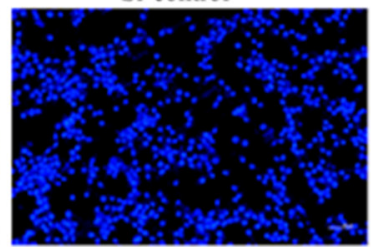

Si-control

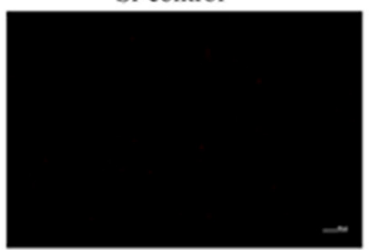

Si-control

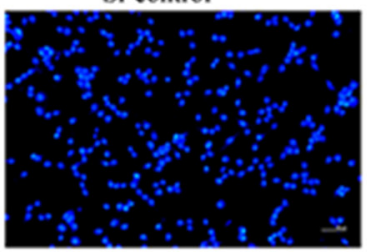

Si-control

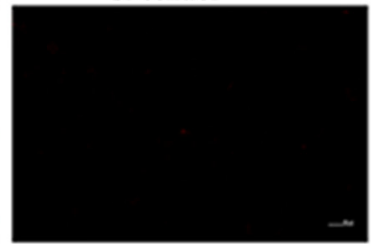

Si-RhoA

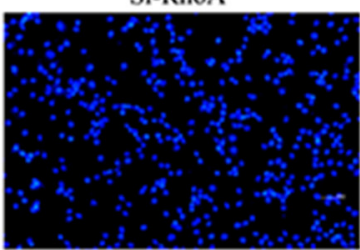

Si-RhoA

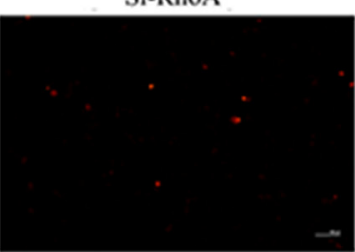

Si-RhoA

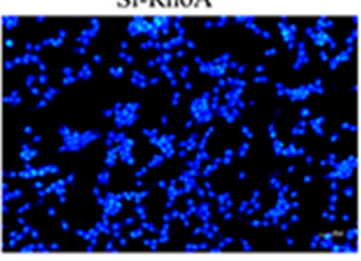

Si-RhoA

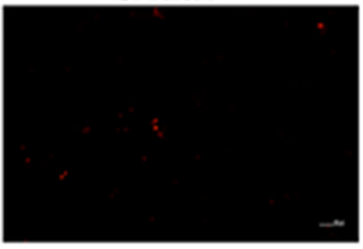

SW620

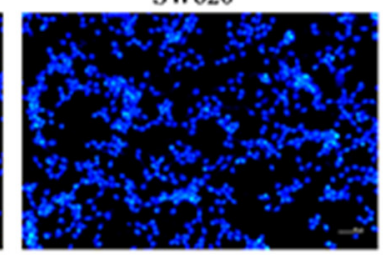

SW620

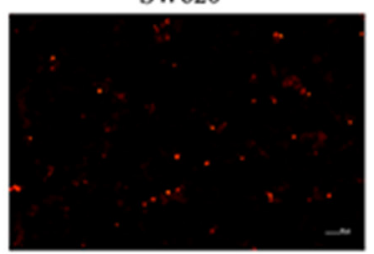

LoVo

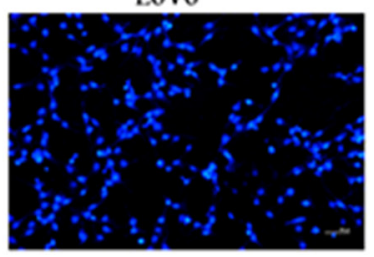

LoVo

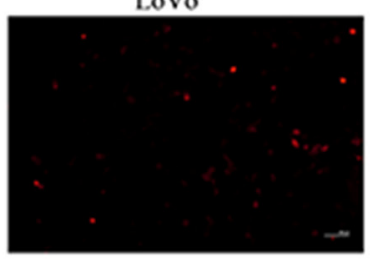

C

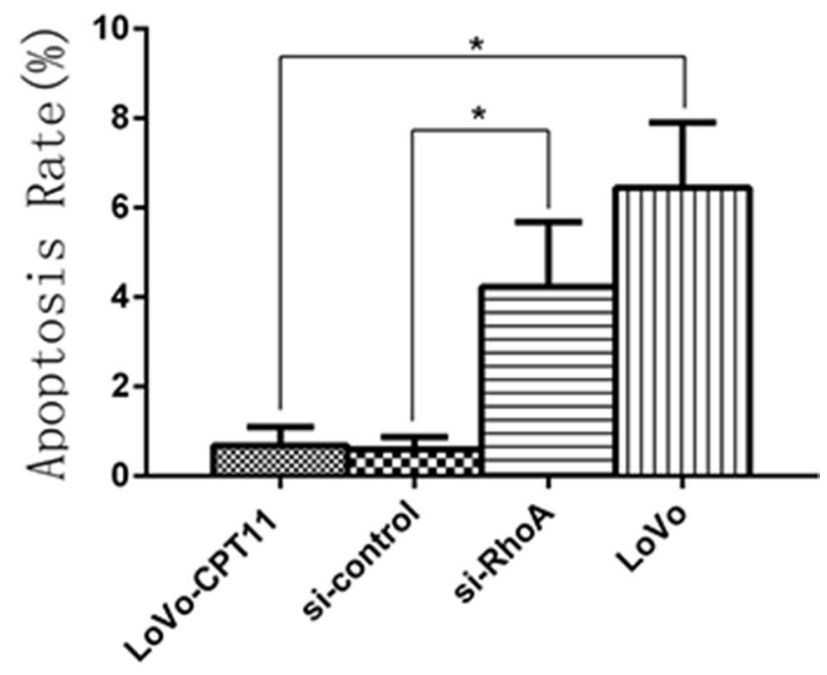

Figure 4: Inhibition of RhoA induces apoptosis in CPT-11-resistant CRC cells by TUNEL assay. (A) The apoptosis rates of CPT-11-resistant CRC cells (SW620/CPT-11 or LoVo/CPT-11), CPT-11-resistant CRC cells transfected with si-control or si-RhoA, and parental cells were determined by TUNEL assay. (B) and (C) Apoptosis rate of CRC cells in A was quantified. Data are representative of three independent experiments. *indicates $p<0.05$. 
In support, our study found increased expression of $\mathrm{ABC}$ transporters, MRP1 and P-gp, in CPT-11-resistant CRC cells. Importantly, suppression of RhoA in CPT-11resistant CRC cells leads to decreased expression of MRP1 and P-gp. We propose that inhibition of RhoA could rescue chemoresistance, at least partially, by dampening $\mathrm{ABC}$ transporter expression in CRC cells.

In addition to increased $\mathrm{ABC}$ transporters, we have further found that suppression of apoptosis is another important mechanism of drug resistance of CRC and that RhoA critically inhibits apoptosis in CPT-11resistant CRC. Studies have shown that Bcl-2 and Bax play important roles in mediating drug-induced apoptosis and drug resistance in various tumor cells, including hepatocellular carcinoma, bladder, lung and ovarian cancer [20-23]. Here we show that RhoA inhibits druginduced apoptosis by impacting on Bcl-2, Bcl-xl and Bax.

Growing evidences support the notion that cancer is a disease driven by cancel stem cells that are responsible for tumor initiation, growth, metastasis, therapy resistance, relapse, and poor prognosis [24-27]. Several studies have demonstrated that the isolated side population (SP) cells from solid tumors exhibit cancer stem cell-like properties, and are responsible for drug resistance during chemotherapy and tumor recurrence [24-27]. In the current study, we have found that SP cells are increased in CPT-11-resistant CRC cells and suppression of RhoA leads to a sharp decrease of SP cells. It indicates that, in addition to membrane transport proteins and survival, cancer stem cells/SP cells are also regulated by RhoA and contribute to drug resistance of CRC.

In conclusion, our study shows that RhoA is involved in chemoresistance of CRC by regulating the expression of membrane transporters, apoptotic proteins and the proportion of SP cells. Thus, the expression of RhoA may indicate a poor prognosis due to the high probability to therapy resistance and, on the other hand, chemoresistance can be compromised through interfering RhoA expression.

\section{MATERIALS AND METHODS}

\section{Cell culture}

All cell lines were maintained in RPMI 1640 medium (Invitrogen, Gaithersburg, MD, USA) supplemented with $10 \%$ heat-inactivated fetal bovine serum (FBS) (Gibco, Gaithersburg, USA), 100 units $/ \mathrm{ml}$

A
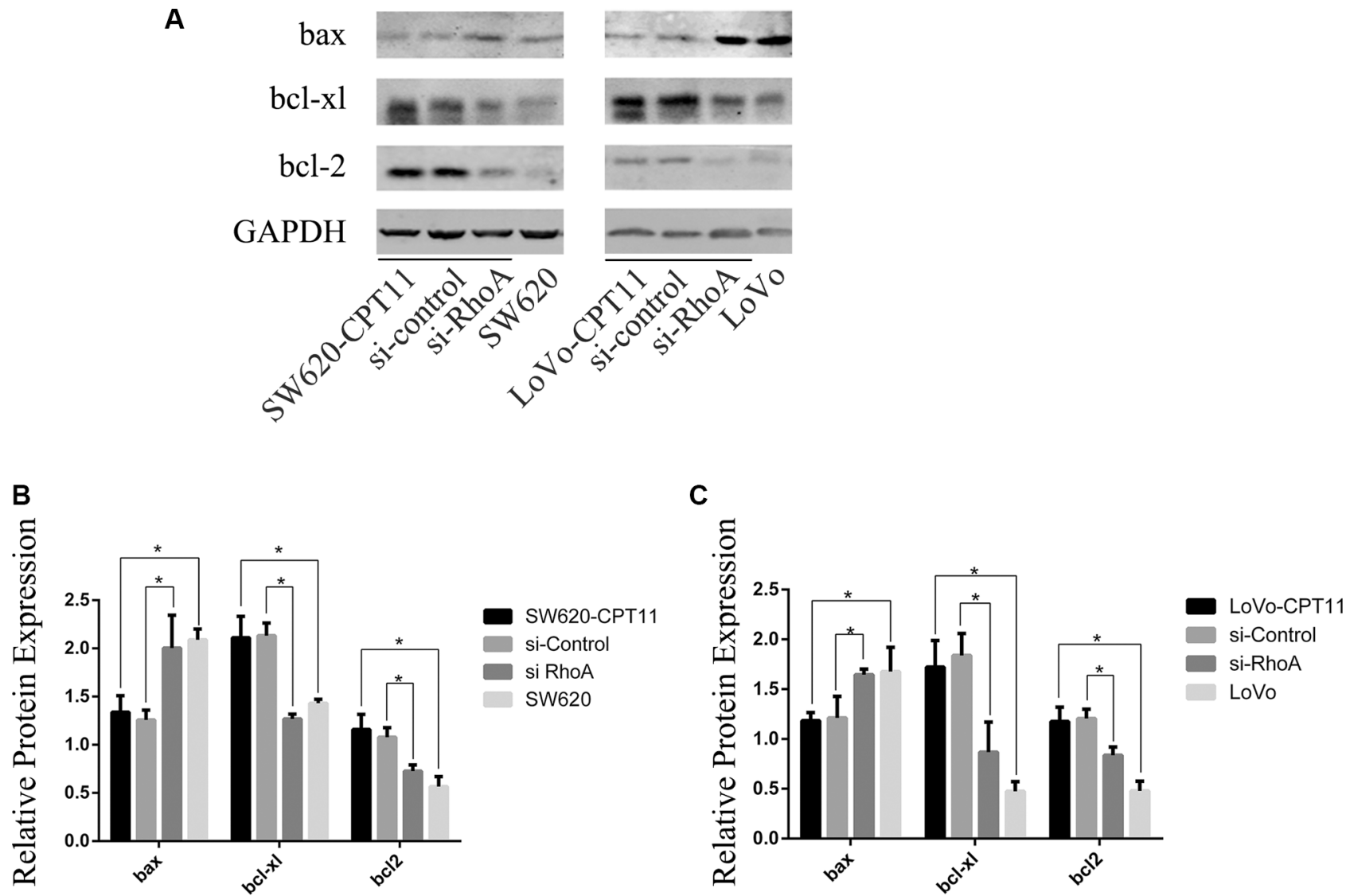

Figure 5: RhoA affects the expression of bcl-2, bcl-xl and bax in CPT-11-resistant CRC cells. (A) Western-blot analysis of CPT-11-resistant CRC cells (SW620/CPT-11 or LoVo/CPT-11), CPT-11-resistant CRC cells transfected with si-control or si-RhoA, and parental cells for bcl-2, bcl-xl and bax protein expression. (B) The band intensities in A were quantified with the ODYSSEY Infrared Imaging System (LI-COR Biosciences). Data represent the mean \pm S.D. from three independent experiments. *indicates $p<0.05$. 
A

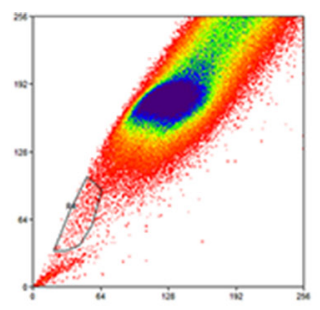

SW620-CPT11(0.13\%)

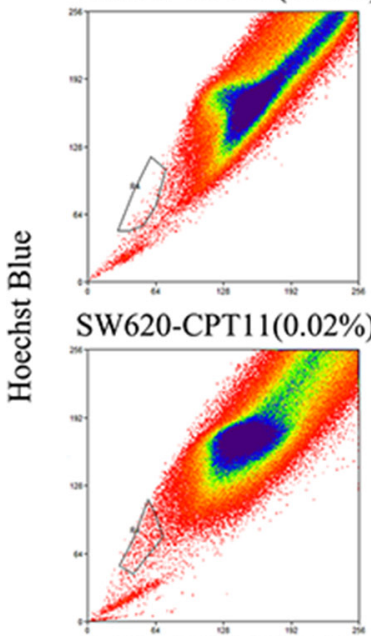

LoVo-CPT11(0.12\%)

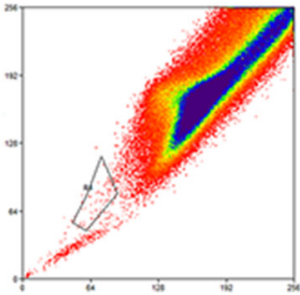

LoVo- CPT11(0.02\%)

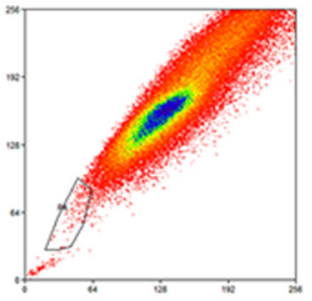

si-control $(0.18 \%)$

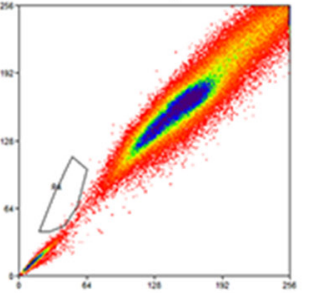

si-control $(0.01 \%)$

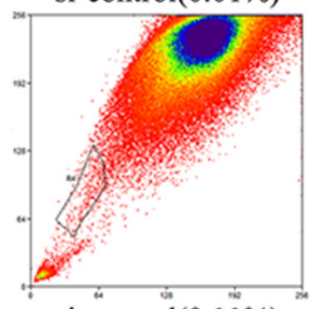

si-control $(0.11 \%)$

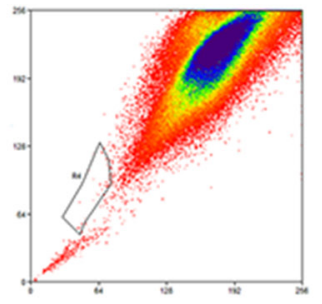

si-Control(0.01\%)

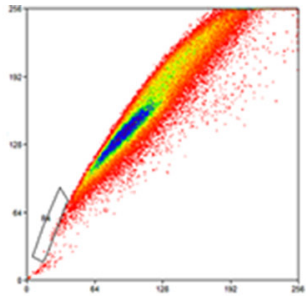

si-RhoA $(0.04 \%)$

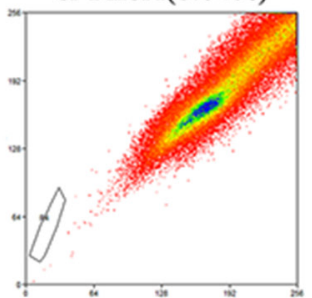

si-RhoA $(0.01 \%)$

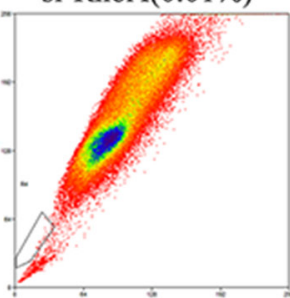

si-RhoA $(0.03 \%)$

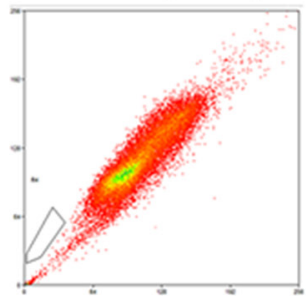

si-RhoA $(0.00 \%)$

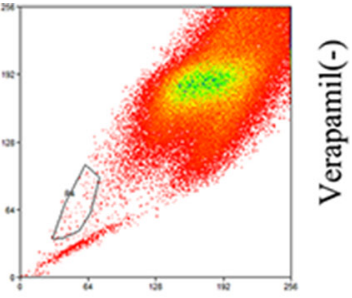

SW620(0.05\%)

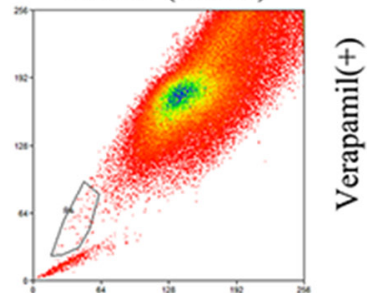

SW620(0.03\%)

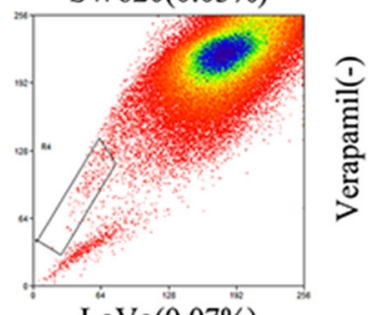

$\operatorname{LoVo}(0.07 \%)$

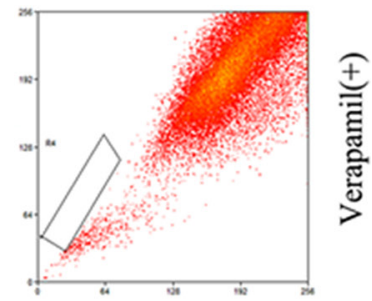

LoVo( $0.01 \%)$

Hoechst Red

B

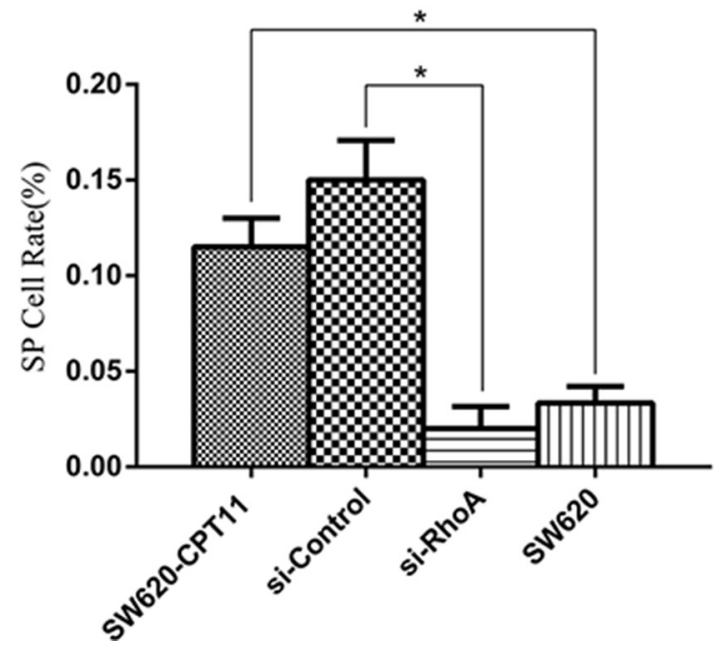

C

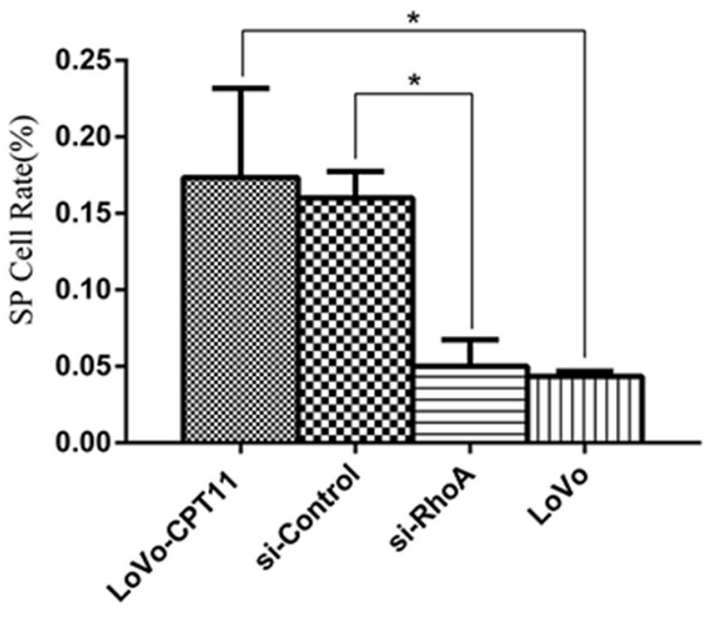

Figure 6: Inhibition of RhoA decreases the proportion of SP cells in CPT-11-resistant CRC cells. (A) The proportion of SP cells was detected using the Hoechst-33342 dye exclusion technique in CPT-11-resistant CRC cells (SW620/CPT-11 or LoVo/CPT-11) and CPT-11-resistant CRC cells transfected with si-control or si-RhoA, and parental cells. (B) and (C) The proportion of SP cells in A was quantified $(p<0.05)$. Data are representative of three independent experiments. *indicates $p<0.05$. 
of penicillin $\mathrm{G}$ sodium, and $100 \mu \mathrm{g} / \mathrm{ml}$ streptomycin sulfate (Sigma, Saint Louis, MO, USA), in a humidified atmosphere containing $5 \% \mathrm{CO}_{2}$ at $37^{\circ} \mathrm{C}$.

\section{RNA extraction and qRT-PCR analysis}

Total RNA was extracted using Trizol (Invitrogen), treated with DNase I (Takara) to eliminate contaminating genomic DNA, and reverse-transcribed into cDNA with the Reverse Transcriptase M-MLV (TaKaRa). Real time PCR was performed using a SYBR Premix Ex TaqTM kit (TaKaRa) on the iQ5 Real-Time PCR Detection System (Bio-Rad, Hercules, CA, USA). PCR primers used were as following: GAPDH FW, 5'-GAAGGTGAAGGTCGGAGT-3' and RV 5'-GAAGAT GGTGATGGGATTTC-3', RhoA FW 5'-GATTGGCGCT TTTGGGTACAT-3' and RV 5'-AGCAGCTCTCGTA GCCATTTC-3'. Expression of RhoA, relative to GAPDH, was determined using the $2^{-\Delta \Delta C \mathrm{CT}}$ method.

\section{Western blot analysis}

Total-cell lysates were prepared using RIPA buffer (150 mM NaCl, 1\%NP-40, $50 \mathrm{mM}$ Tris- $\mathrm{HCl}$ (pH 7.4), $1 \mathrm{mM}$ phenylmethylsulfonyl fluoride, $1 \mu \mathrm{g} / \mathrm{ml}$ leupeptin, $1 \mathrm{mM}$ Deoxycholic acid and $1 \mathrm{mM}$ EDTA) containing a cocktail of protease inhibitors and phosphatase inhibitors (Calbiochem, Darmstadt, Germany). Equal amount of proteins $(40 \sim 60 \mu \mathrm{g})$ was separated by $12 \%$ SDS-PAGE and transferred to PVDF membrane (Millipore, Bedford, MA, USA) using the Bio-Rad semidry transfer system. The following antibodies were used for Western blot: Anti-RhoA antibody (Santa Cruz Biotechnologies, USA), Anti-GAPDH antibody, Anti-bcl2 antibody, Anti-bcl-xl antibody, Anti-bax antibody (Cell Signaling Technology, USA), Anti-ABCB1 antibody, Anti-ABCC1 antibody, Anti-GSPT1 antibody (Abcam, UK). Blotted proteins were detected and quantified using the ODYSSEY Infrared Imaging System (LI-COR Biosciences, Lincoln, NE, USA).

\section{Transfection}

Control siRNA and RhoA-siRNA targeting RhoA coding sequences (5'-UGAGCAAGCAUGUCUUUCCAC AGGC-3', 5'-GCCUGUGGAAAGACAUGCUUGCUCA$3^{\prime}$ ) were synthesized by RiboBio (RiboBio, Guangzhou, China). RNA oligonucleotides were transfected into cells using Lipofectamine 2000 (Invitrogen) according to the manufacturer's protocol.

\section{In vitro drug sensitivity assay}

Cells were seeded onto 96-well culture plates at a density $1.0 \times 10^{3} /$ well with RPMI1640 100 ul per well. A total of 4 groups were set in this study: CPT-11 resistant group, siRNA control group, RhoA siRNA group, parental colorectal cancer cell group. Chemotherapeutic drugs CPT-11, L-OHP, PTX, VP16, EPI, DDP, 5-FU (Dalian Meilun Biology Technology co., LTD, China) were added at increasing concentration for 24 hours. The cells were continuously incubated for 48 hours after removing the chemotherapeutic drugs. In vitro drug sensitivity was then determined using Cell Counting Kit- 8 assay (Dojindo Molecular Technologies, Inc.Japan). In brief, the cells were added of $10 \mathrm{ul}$ of CCK-8, incubated for three hours, measured for the absorbance at $450 \mathrm{~nm}$, and calculated for the inhibitory concentration of $50 \%$ cells $\left(\mathrm{IC}_{50}\right)$. The assays were conducted in triplicate and repeated at least 3 times.

\section{Flow cytometry}

$1 \times 10^{6}$ cells were collected by centrifugation and resuspended using $500 \mathrm{ul}$ bonding buffer (KeyGen Biotechnology Co., Ltd., China). $5 \mu \mathrm{l}$ of Annexin V-FITC and $5 \mu \mathrm{l}$ of propidium iodide were added into the collected cells (KeyGen Biotechnology Co., Ltd., China) and incubated for 5 minutes at room temperature in the dark. Annexin V-FITC binding was analyzed by flow cytometry $(E x=488 \mathrm{~nm} ; \mathrm{Em}=530 \mathrm{~nm})$ using FITC signal detector and PI staining by the phycoerythrin emission signal detector ( Beckman Coulter, Inc., USA). The assays were conducted in triplicate and repeated at least 3 times.

\section{TUNEL assay}

TUNEL assay is performed with a kit from Millipore according to the manufacturer's instructions and observed by a fluorescence microscopy (Nikon, Japan). The assays were conducted in triplicate and repeated at least 3 times.

\section{SP analysis}

The cells were harvested and resuspended at $1 \times 10^{6} / \mathrm{ml}$ in prewarmed DMEM (Life technologies, USA) containing $2 \%$ fetal calf serum (Kang Yuan Biology, China) and 10 mM HEPES (Dalian Meilun Biology Technology co., LTD, China).The cell suspension was gently mixed in $15 \mathrm{ml}$ polypropylene centrifuge tube (Corning, USA). And then Hoechst 33342 (Invirtrogen, USA) was added to a final concentration of $5 \mathrm{ug} / \mathrm{ml}$ and verapamil (SigmaAldrich, USA) of $80 \mathrm{ug} / \mathrm{ml}$ to control group. The cells were incubated for 90 minutes at $37^{\circ} \mathrm{C}$ and mixed every 30 minutes. After incubation, the cells were centrifuged at $500 \mathrm{~g}$ for 5 minutes, and resuspended in cold HBSS. Propidium iodide (Sigma-Aldrich,USA) was added into each tube at concentration of $1 \mathrm{ug} / \mathrm{ml}$ to discriminate dead cells. In order to avoid the effluxion of Hoechst 33342, further tests were performed at $4^{\circ} \mathrm{C}$. SP cells were tested using flow cytometry and analyzed using Summit 5.2 software (Beckman Coulter, Inc., USA). The assays were conducted in triplicate and repeated at least 3 times. 


\section{Statistical analysis}

$\mathrm{IC}_{50}$ was calculated using probit regression analysis. The results were reported as Mean \pm SD and analyzed by student $t$-test or one way ANOVA by SPSS 18.0 analytic software. $P$ values of $<0.05$ were considered as statistical significant.

\section{Abbreviations}

RhoA, ras homolog gene family, member A; siRhoA, siRNA targeting RhoA coding sequences; mCRC, metastatic colorectal cancer; CPT-11, irinotecan; MDR1, multidrug resistance 1; MRP1, multi-drug resistant associate protein; L-OHP, oxaliplatin; DDP, cisplatin; PTX, paclitaxel; 5-FU, 5-fluorouracil; EPI, epirubicin; VP-16, etoposide.

\section{ACKNOWLEDGMENTS AND FUNDING}

This work was supported by National Natural Science Foundation of China (Grant No. 81572850 \& 31200975) and Outstanding Youth Foundation of Sichuan University (Grant No. 2013SCU04A18).

\section{CONFLICTS OF INTEREST}

The authors have no conflicts of interests.

\section{REFRENCES}

1. Siegel R, Desantis C, Jemal A. Colorectal cancer statistics. Cancer J Clin. 2014; 64:104-17.

2. Wilkinson N, Scott-Conner CE. Surgical Therapy for Colorectal Adenocarcinoma. Gastroenterol Clin North Am. 2008; 37:253-267.

3. Dodds HM, Haaz MC, Riou JF, Robert J, Rivory LP. Identification of a new metabolite of CPT-11 (irinotecan): pharmacological properties and activation to $\mathrm{SN}-38$. J Pharmacol Exp Ther. 1998; 286:578-83.

4. Saltz LB, Douillard JY, Pirotta N, Alakl M, Gruia G, Awad L, Elfring GL, Locker PK, Miller LL. Irinotecan plusfluorouracil/leucovorin for metastatic colorectal cancer: a new survival standard. Oncologist. 2001; 6:81-91.

5. Szakács G, Annereau JP, Lababidi S, Shankavaram U, Arciello A, Bussey KJ, Reinhold W, Guo Y, Kruh GD, Reimers M, Weinstein JN, Gottesman MM. Predicting drug sensitivity and resistance: profiling $\mathrm{ABC}$ transporter genes in cancer cells. Cancer Cell. 2004; 6:129-137.

6. Gillet JP, Efferth T, Steinbach D, Hamels J, de Longueville F, Bertholet V, Remacle J. Microarray-based detection of multidrugresistance in human tumor cells by expression profiling ofATP-binding cassette transporter genes, Cancer Res. 2004; 64:8987-8993.
7. Cory S, Adams JM. The Bcl 2 family: regulators of the cellular life-or-deathswitch, Nat Rev Cancer. 2002; 2:647-656.

8. Brenner C, Cadiou H, Vieira HL, Zamzami N, Marzo I, Xie Z, Leber B, Andrews D, Duclohier H, Reed JC, Kroemer G. Bcl-2 and Bax regulate the channelactivity of the mitochondrial adenine nucleotide translocator. Oncogene. 2000; 19:329-336.

9. Chen ZX, Pervaiz S. Bcl-2 induces pro-oxidant state by engaging mitochondrialrespiration in tumor cells. Cell Death Differ. 2007; 14:1617-1627.

10. Chen L, Gilkes DM, Pan Y, Lane WS, Chen J. ATM and Chk2-dependentphosphorylation of MDMX contribute to p53 activation after DNA damage. EMBO J. 2005; 24:3411-3422.

11. Sau A, Pellizzari Tregno F, Valentino F, Federici G, Caccuri AM. Glutathione S-transferases and development of new principles to overcome drug resistance. Arch Biochem Biophys. 2010; 500:116-122.

12. Huang Y, Sadée W. Membrane transporters and channels in chemoresistance and -sensitivity of tumor cells. Cancer Letters. 2006; 239:168-182.

13. Liu M, Bi F, Zhou X, Zheng Y. Rho GTPase regulation by miRNAs and covalent modifications. Trends Cell Biol. $2012 ; 22: 365-373$.

14. Etienne-Manneville S, Hall A. Rho GTPases in cell biology. Nature. 2002; 420:629-35.

15. Sahai E, Marshall CJ. RHO-GTPases and cancer. Nat Rev Cancer. 2002; 2:133-42.

16. Gong FM1, Peng XC, Tan BX, Ge J, Chen X, Chen Y, Xu F, Bi F, Hou JM, Liu JY. Comparative proteomic analysis of irinotecan-sensitive colorectal carcinoma cell line and its chemoresistant counterpart. Anticancer Drugs. 2011; 22:500-6.

17. Peng XC, Gong FM, Wei M, Chen X, Chen Y, Cheng K, Gao F, Xu F, Bi F, Liu JY. Proteomic analysis of cell lines to identify the irinotecan resistance proteins. Biosci. 2010; 35:557-64

18. Ishii $\mathrm{T}$, Teramoto $\mathrm{S}$, Matsuse $\mathrm{T}$. GSTP1 affects chemoresistance against camptothecin in human lung adenocarcinoma cells. Cancer Letters. 2004; 216:89-102.

19. Moserle L, Ghisi M, Amadori A, Indraccolo S. Side population and cancer stem cells: Therapeutic implications, Cancer Letters. 288:1-9.

20. Ye D, Li H, Qian S, Sun Y, Zheng J, Ma Y. bcl-2/ bax expression and p53 gene status in human bladder cancer: relationship to early recurrence with intravesical chemotherapy after resection. J Urol. 1998; 160:2025-2029.

21. Han JY, Chung YJ, Park SW, Kim JS, Rhyu MG, Kim HK, Lee KS. The relationship between cisplatin-induced apoptosis and p53, bcl-2 and bax expression in human lung cancer cells, Korean J Intern Med. 1999; 14:42-52.

22. Stojcheva N, Schechtmann G, Sass S, Roth P, Florea AM, Stefanski A, Stühler K, Wolter M, Müller NS, Theis FJ, 
Weller M, Reifenberger G, Happold C. MicroRNA-138 promotes acquired alkylator resistance in glioblastoma by targeting the Bcl-2-interacting mediator BIM. Oncotarget. 2016; 7:12937-50. doi: 10.18632/oncotarget.7346.

23. Murata T, Haisa M, Uetsuka H, Nobuhisa T, Ookawa T, Tabuchi Y, Shirakawa Y, Yamatsuji T, Matsuoka J, Nishiyama M, Tanaka N, Naomoto Y. Molecular mechanism of chemoresistance to cisplatin in ovarian cancer cell lines. Int J Mol Med. 2004; 13:865-868.

24. Hu L1, McArthur C, Jaffe RB. Ovarian cancer stem-like side-population cells are tumourigenic and chemoresistant. Br J Cancer. 2010; 102:1276-1283.

25. Dean M, Fojo T, Bates S. Tumour stem cells and drug resistance. Nat Rev Cancer. 2005; 5:275-84.
26. Zhang X, Cheng X, Lai Y, Zhou Y, Cao W, Hua ZC. Salmonella VNP20009-mediated RNA interference of ABCB5 moderated chemoresistance of melanoma stem cell and suppressed tumor growth more potently. Oncotarget. 2016; 7:14940-50. doi: 10.18632/oncotarget.7496.

27. Hirschmann-Jax C, Foster AE, Wulf GG, Nuchtern JG, Jax TW, Gobel U, Goodell MA, Brenner MK. A distinct "side population" of cells with high drug efflux capacityinhuman tumor cells. Proc Natl Acad Sci USA. 2004; 101:14228-33.

28. Carloni V, Mazzocca A, Mello T, Galli A, Capaccioli S. Cell fusion promotes chemoresistance in metastatic colon carcinoma. Oncogene. 2013; 32:2649-60. 